\title{
Numerical solution of a parabolic equation on the sphere using Laplace transforms and radial basis functions
}

\author{
Quoc Thong Le Gia ${ }^{1} \quad$ William McLean ${ }^{2}$
}

(Received 27 January 2011; revised 20 April 2011)

\begin{abstract}
We propose a method to construct numerical solutions of a parabolic equation on the unit sphere. The time discretisation uses Laplace transformation and quadrature. The spatial approximation employs radial basis functions restricted to the sphere. The method provides a parallel algorithm to construct high accuracy numerical solutions.
\end{abstract}

\section{Contents}

1 Introduction

C90

2 Preliminaries

C91

2.1 Resolvent estimates . . . . . . . . . . . . . . . C91

2.2 Spherical radial basis functions . . . . . . . . . . C93

http://anziamj.austms.org.au/ojs/index.php/ANZIAMJ/article/view/3922 gives this article, (c) Austral. Mathematical Soc. 2011. Published May 6, 2011. ISSN 1446-8735. (Print two pages per sheet of paper.) Copies of this article must not be made otherwise available on the internet; instead link directly to this URL for this article. 
3 The numerical method

C95

3.1 Time discretisation . . . . . . . . . . . . C95

3.2 Galerkin approximation by radial basis functions . . . . . C96

3.3 Fully-discrete solution . . . . . . . . . . . . . C97

4 Numerical experiments

C97

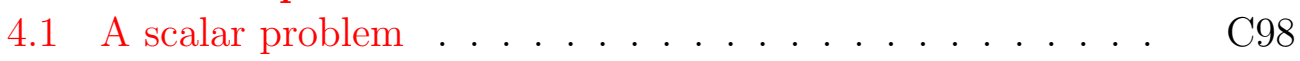

4.2 A problem on the unit sphere . . . . . . . . . . C99

References

C101

\section{Introduction}

Let $\mathbb{S}^{n}:=\left\{\boldsymbol{x} \in \mathbb{R}^{\mathfrak{n}+1}:|\boldsymbol{x}|=1\right\}$ denote the $\mathrm{n}$-dimensional unit sphere, with $n \geqslant 2$. We consider a parabolic equation on $\mathbb{S}^{n}$,

$$
\partial_{t} u+A u=f(t), \quad \text { for } t>0, \quad \text { with } u(0)=u_{0},
$$

where $\partial_{t}=\partial / \partial t$ and $A$ is a linear, self-adjoint, second order, strongly elliptic, partial differential operator on $\mathbb{S}^{n}$. The coefficients of $A$ must be independent of $t$, and later we restrict our attention to the case when $-A$ is the LaplaceBeltrami operator. In that case, equation (1) describes the diffusion of heat on the surface of the sphere with a given density $f$ of sources $[2,4]$.

Denoting the Laplace transform of $u$ with respect to $t$ by

$$
\widehat{u}(z)=\mathcal{L}\{u(t)\}:=\int_{0}^{\infty} e^{-z t} u(t) d t,
$$

we find that the solution of (1) formally satisfies

$$
(z \mathrm{I}+\mathrm{A}) \hat{\mathfrak{u}}(z)=g(z):=\mathfrak{u}_{0}+\hat{\mathrm{f}}(z),
$$

where I denotes the identity operator. Assuming that the operator $(z I+A)$ is invertible, we write

$$
\widehat{u}(z)=(z I+A)^{-1} g(z),
$$


and seek to recover $\mathfrak{u}(\mathbf{t})$ via the inversion formula for the Laplace transform,

$$
\mathfrak{u}(\mathrm{t})=\frac{1}{2 \pi \mathfrak{i}} \int_{\Gamma_{0}} e^{z \mathfrak{t}} \hat{u}(z) d z, \quad \text { for } t>0,
$$

where $\Gamma_{0}$ is the line $\mathfrak{R} z=\omega$, with any $\omega>0$, and $\mathfrak{I} z$ increasing.

Instead of using time stepping [2], our approach here is based on an approximation by quadrature of the representation (5), with an appropriately deformed contour of integration. This idea was introduced by Sheen, Sloan and Thomée [7] for a parabolic problem on a bounded domain $\Omega$ in $\mathbb{R}^{n}$, and used in conjunction with a spatial discretisation by finite elements. McLean and Thomée have refined the original error analysis in subsequent articles [3]. The novel feature in this article is the use of spherical radial basis functions instead of finite elements. Our aim here is to demonstrate the practical effectiveness of the numerical method; we defer a complete error analysis to a subsequent article.

\section{Preliminaries}

\subsection{Resolvent estimates}

Our assumptions on the operator $A$ ensure the existence of a complete orthonormal system of eigenfunctions $\psi_{1}, \psi_{2}, \psi_{3}, \ldots$, with corresponding real eigenvalues $\lambda_{1}, \lambda_{2}, \lambda_{3}, \ldots$ Thus, $A \psi_{j}=\lambda_{j} \psi_{j}$ for all $\mathfrak{j}$, and each $\mathfrak{u} \in \mathrm{L}_{2}\left(\mathbb{S}^{n}\right)$ has an eigenfunction expansion of the form

$$
u=\sum_{j=1}^{\infty}\left\langle u, \psi_{j}\right\rangle \psi_{j} .
$$

We make the additional assumption that all the eigenvalues are non-negative, so that the initial value problem (1) is well-posed, and the resolvent $(z I-A)^{-1}$ 
exists for $z$ outside any sector

$$
\Sigma_{\varphi}=\{z \in \mathbb{C}: z \neq 0 \text { and }|\arg z| \leqslant \varphi\} \cup\{0\}, \quad \text { with } 0<\varphi<\pi / 2 .
$$

Hence, $(z \mathrm{I}+\mathrm{A})^{-1}$ exists for $z$ inside $\Sigma_{\pi-\varphi}$. The following estimates hold for a much wider class of sectorial opertors, but in our case we give an elementary and self-contained proof.

Lemma 1 Given $\varphi$ satisfying $0<\varphi<\pi / 2$, there exists a constant $M>0$ such that

$$
\left\|(z \mathrm{I}+\mathrm{A})^{-1}\right\| \leqslant \frac{M}{|z|}, \quad \text { for } z \in \Sigma_{\pi-\varphi} .
$$

If, in addition to the assumptions above, 0 is not an eigenvalue of $\mathrm{A}$, then there exists a constant $M^{\prime}$ such that

$$
\left\|(z \mathrm{I}+\mathrm{A})^{-1}\right\| \leqslant \frac{M^{\prime}}{1+|z|}, \quad \text { for } z \in \Sigma_{\pi-\varphi} .
$$

Proof: Let $z=\rho e^{i \theta}$ with $|\theta|<\pi-\varphi$ and $\rho>0$. If $0<|\theta| \leqslant \pi / 2$, then $0 \leqslant \mathfrak{R} z \leqslant \mathfrak{R}\left(z+\lambda_{\mathrm{j}}\right)$ and $\mathfrak{I}(z)=\mathfrak{I}\left(z+\lambda_{\mathrm{j}}\right)$, so $|z| \leqslant\left|z+\lambda_{\mathrm{j}}\right|$. If $\pi / 2<|\theta|<\pi-\varphi$, then $|z| \sin \varphi \leqslant \rho \sin |\theta|=|\Im z|=\left|\Im\left(z+\lambda_{j}\right)\right| \leqslant\left|z+\lambda_{j}\right|$, and thus

$$
\frac{1}{\left|z+\lambda_{j}\right|} \leqslant \frac{1}{|z| \sin \varphi}, \quad \text { for } z \in \Sigma_{\pi-\varphi} .
$$

The eigenfunction expansion (6) shows that

$$
\left\|(z \mathrm{I}+\mathrm{A})^{-1} \mathrm{u}\right\|^{2}=\sum_{j=1}^{\infty} \frac{\left|\left\langle\mathrm{u}, \psi_{\mathrm{j}}\right\rangle\right|^{2}}{\left|z+\lambda_{j}\right|^{2}} \leqslant \frac{1}{(|z| \sin \varphi)^{2}} \sum_{j=1}^{\infty}\left|\left\langle u, \psi_{j}\right\rangle\right|^{2}=\left(\frac{\|u\|}{|z| \sin \varphi}\right)^{2}
$$

for any $u \in L_{2}\left(\mathbb{S}^{n}\right)$, so the estimate (7) holds with $M=1 / \sin \varphi$.

We relabel the eigenvalues, if necessary, so that $\lambda_{1} \leqslant \lambda_{2} \leqslant \lambda_{3} \leqslant \cdots$ with $\lambda_{j} \rightarrow$ $\infty$ as $j \rightarrow \infty$, and assume $\lambda_{1}>0$. If $|z|<\lambda_{1} / 2$ then $\left|z+\lambda_{1}\right| \geqslant \lambda_{1}-|z| \geqslant \lambda_{1} / 2$ and hence

$$
\frac{1+|z|}{\left|z+\lambda_{1}\right|} \leqslant \frac{1+\lambda_{1} / 2}{\lambda_{1} / 2}=1+\frac{2}{\lambda_{1}} .
$$


If $|z| \geqslant \lambda_{1} / 2$ with $|\arg z|<\pi-\varphi$, then $1+|z| \leqslant\left(2 / \lambda_{1}\right)|z|+|z|$ and hence

$$
\frac{1+|z|}{\left|z+\lambda_{j}\right|} \leqslant \frac{1+|z|}{|z| \sin \varphi} \leqslant \frac{1}{\sin \varphi}\left(1+\frac{2}{\lambda_{1}}\right) .
$$

By reasoning as in (9), we establish (8) with $M^{\prime}=\left(1+2 / \lambda_{1}\right) / \sin \varphi$.

We now fix an angle $\bar{\beta}$ in the range $\pi / 2<\bar{\beta}<\pi$, and make the assumption that $\hat{f}(z)$ admits a bounded analytic continuation to a sector $\omega+\Sigma_{\beta} \subseteq \Sigma_{\bar{\beta}}$ with $\omega \geqslant 0$ and $\pi / 2<\beta \leqslant \bar{\beta}$. It then follows that (4) defines $\hat{u}(z)$ as an analytic function in $\omega+\Sigma_{\beta}$.

\subsection{Spherical radial basis functions}

We assume that $\Phi: \mathbb{S}^{n} \times \mathbb{S}^{n} \rightarrow \mathbb{R}$ is a strictly positive-definite kernel on $\mathbb{S}^{n}$, that is $[6,9]$,

1. $\Phi$ is continuous;

2. $\Phi(\boldsymbol{x}, \mathbf{y})=\Phi(\mathbf{y}, \boldsymbol{x})$ for all $\boldsymbol{x}, \boldsymbol{y} \in \mathbb{S}^{n}$;

3. For any set of distinct points $X=\left\{\boldsymbol{x}_{1}, \boldsymbol{x}_{2}, \ldots, \boldsymbol{x}_{K}\right\} \subset \mathbb{S}^{n}$, the $K \times K$ matrix $\left[\Phi\left(\boldsymbol{x}_{\mathfrak{p}}, \boldsymbol{x}_{\mathfrak{q}}\right)\right]$ is positive definite.

The uniformity of the point set $\mathrm{X}$ is measured by its mesh norm $\mathrm{h}=\mathrm{h}_{\mathrm{X}}$ and its separation radius $\mathrm{r}=\mathrm{r}_{\mathrm{X}}$, defined by

$$
h_{X}:=\sup _{\mathbf{y} \in \mathbb{S}^{n}} \min _{\boldsymbol{x} \in X} \cos ^{-1}(\mathbf{y} \cdot \boldsymbol{x}) \quad \text { and } \quad r_{X}:=\frac{1}{2} \min _{\substack{\boldsymbol{x}, \boldsymbol{y} \in X \\ \boldsymbol{x} \neq \mathbf{y}}} \cos ^{-1}(\mathbf{y} \cdot \boldsymbol{x}) .
$$

In words, $h$ is the maximum geodesic distance from a point on $\mathbb{S}^{n}$ to the nearest point of $X$. Together, $\Phi$ and $X$ determine a space of spherical radial basis functions (RBFs) on $\mathbb{S}^{n}$,

$$
V_{h}:=\operatorname{span}\left\{\Phi_{p}: 1 \leqslant p \leqslant K\right\}, \quad \text { where } \Phi_{p}(x):=\Phi\left(x_{p}, x\right) .
$$


TABLE 1: Compactly-supported radial basis functions.

\begin{tabular}{cccc}
\hline$m$ & $\rho_{m}(r)$ & Smoothness & $\tau$ \\
\hline 2 & $(1-r)_{+}^{6}\left(3+18 r+35 r^{2}\right)$ & $C^{4}\left(\mathbb{R}^{3}\right)$ & $7 / 2$ \\
3 & $(1-r)_{+}^{8}\left(1+8 r+25 r^{2}+32 r^{3}\right)$ & $C^{6}\left(\mathbb{R}^{3}\right)$ & $9 / 2$ \\
\hline
\end{tabular}

We consider a kernel $\Phi$ of the form

$$
\Phi(\boldsymbol{x}, \mathbf{y})=\phi(\boldsymbol{x} \cdot \mathbf{y}), \quad \text { for } \boldsymbol{x}, \boldsymbol{y} \in \mathbb{S}^{n}
$$

and expand the univariate function $\phi:[-1,1] \rightarrow \mathbb{R}$ in a Fourier-Legendre series,

$$
\phi(t)=\sum_{\ell=0}^{\infty} \hat{\phi}_{\ell} P_{\ell}(t), \quad \text { with } \hat{\phi}_{\ell}=\int_{-1}^{1} P_{\ell}(t) \phi(t)\left(1-t^{2}\right)^{(n-2) / 2} d t
$$

where the Legendre polynomials $P_{0}, P_{1}, P_{2}, \ldots$ are scaled to make them orthonormal with respect to the weight $\left(1-t^{2}\right)^{(n-2) / 2}$ over the interval $(-1,1)$. Chen et al. [1] established a complete characterisation of strictly positive definite kernels on $\mathbb{S}^{n}$ : the kernel $\Phi$ is strictly positive definite if and only if $\hat{\phi}_{\ell} \geqslant 0$ for all $\ell \geqslant 0$ and $\hat{\phi}_{\ell}>0$ for infinitely many even values of $\ell$ and infinitely many odd values of $\ell$.

We assume simply that $\hat{\phi}_{\ell}>0$ for all $\ell \geqslant 0$. In addition, we require that

$$
\hat{\phi}_{\ell}=\mathcal{O}\left(\ell^{-2 \tau}\right) \text { as } \ell \rightarrow \infty, \quad \text { for some } \tau>\mathrm{n} / 2,
$$

thereby ensuring that $\Phi$ is continuous on $\mathbb{S}^{n} \times \mathbb{S}^{n}$, and that each radial basis function $\Phi_{p}$ belongs to the Sobolev space $H^{\tau}\left(\mathbb{S}^{n}\right)$. For example, the compactly supported radial basis functions introduced by Wendland [8] satisfy these assumption with a strictly positive-definite kernel of the form $\Phi(\boldsymbol{x}, \mathbf{y})=$ $\rho_{\mathfrak{m}}(\sqrt{2-2 x \cdot y})$. Table 1 lists $\rho_{2}$ and $\rho_{3}$ explicitly, along with the values of the exponent $\tau$ in (12). 


\section{The numerical method}

\subsection{Time discretisation}

We define $\Gamma$ to be the parametric curve in the complex plane,

$$
z(\xi)=\omega+\lambda(1-\sin (\delta-i \xi)), \quad \text { for } \xi \in \mathbb{R},
$$

where the constants $\lambda$ and $\delta$ satisfy

$$
\lambda>0 \text { and } 0<\delta<\beta-\frac{\pi}{2} \text {. }
$$

Writing $z=x+i y$, we find that $\Gamma$ is the left branch of the hyperbola

$$
\left(\frac{x-\omega-\lambda}{\lambda \sin \delta}\right)^{2}-\left(\frac{y}{\lambda \cos \delta}\right)^{2}=1
$$

which cuts the real axis at the point $z(0)=\omega+\lambda(1-\sin \delta)$ and has asymptotes $y= \pm(x-\omega-\lambda) \cot \delta$. Thus, the conditions (14) ensure that $\Gamma$ lies in the sector $\omega+\Sigma_{\beta}$ and crosses into the left half-plane. It follows that $\Gamma$ is homotopic with the contour $\Gamma_{0}$ in (5), and so

$$
u(t)=\frac{1}{2 \pi i} \int_{\Gamma} e^{z t} \hat{u}(z) d z, \quad \text { for } t>0 .
$$

Using (13) in (15), we may represent $\mathfrak{u}(\mathbf{t})$ as an integral with respect to $\xi$,

$$
u(t)=\int_{-\infty}^{\infty} v(\xi, t) d \xi, \quad \text { where } v(\xi, t):=\frac{1}{2 \pi i} e^{z(\xi) t} \hat{u}(z(\xi)) z^{\prime}(\xi) .
$$

Since $\left|e^{z(\xi) t}\right|=e^{\Re z(\xi) t}=e^{\omega t} e^{\lambda t(1-\sin \delta \cosh \xi)}$, the integrand exhibits a double exponential decay as $|\xi| \rightarrow \infty$, for any fixed $t>0$. We therefore achieve spectral accuracy using a simple, equal-weight quadrature rule of the form

$$
\mathrm{Q}_{N}(v):=\mathrm{k} \sum_{j=-N}^{N} v\left(\xi_{j}\right) \approx \int_{-\infty}^{\infty} v(\xi) \mathrm{d} \xi, \quad \text { with } \xi_{j}:=j k
$$


for an appropriate choice of the quadrature step $k>0$. Setting $z_{j}:=z\left(\xi_{j}\right)$ and $z_{j}^{\prime}:=z^{\prime}\left(\xi_{j}\right)$, we obtain an approximate solution to our problem (1) of the form

$$
u(t) \approx u_{N}(t):=Q_{N}(v(\cdot, t))=\frac{k}{2 \pi i} \sum_{j=-N}^{N} e^{z_{j} t} \hat{u}\left(z_{j}\right) z_{j}^{\prime} .
$$

To compute $\mathrm{U}_{\mathrm{N}}(\mathrm{t})$ we must solve the $2 \mathrm{~N}+1$ elliptic equations

$$
\left(z_{j} I+A\right) \hat{u}\left(z_{j}\right)=g\left(z_{j}\right), \quad \text { for }|j| \leqslant N .
$$

These equations are independent and hence may be solved in parallel.

Although the $\widehat{u}\left(z_{j}\right)$ determine $U_{N}(t)$ for all $t>0$, in practice the approximation (18) is good only for $t$ in a bounded interval depending on $k$ and the parameters defining the contour $\Gamma$. By Lemma 1, the error analysis by McLean and Thomée [3, Theorem 3.1] applies, so given $\mathrm{N}$ and a time scale $\mathrm{T}$, we can choose $\lambda \propto N / T$ and $k \propto 1 / N$ such that

$$
\left\|\mathrm{U}_{\mathrm{N}}(\mathrm{t})-\mathrm{u}(\mathrm{t})\right\|=\mathrm{O}\left(\mathrm{e}^{-\mu \mathrm{N}}\right), \quad \text { for } \frac{1}{2} \mathrm{~T} \leqslant \mathrm{t} \leqslant 2 \mathrm{~T},
$$

with $\mu>0$ independent of $\mathrm{N}$ and $\mathrm{T}$.

\subsection{Galerkin approximation by radial basis functions}

From now on, we assume that $A=-\Delta^{*}$ in (1), where $\Delta^{*}$ is the LaplaceBeltrami operator on the unit sphere $\mathbb{S}^{n}$. Thus,

$$
\left\langle-\Delta^{*} v, w\right\rangle=\langle\operatorname{grad} v, \operatorname{grad} w\rangle, \quad \text { for } v, w \in \mathrm{H}^{1}\left(\mathbb{S}^{n}\right),
$$

where $\operatorname{grad} v$ denotes the surface gradient of $v$ on $\mathbb{S}^{n}$. In the weak formulation of the elliptic problem (3), we seek $\widehat{u}(z) \in \mathrm{H}^{1}\left(\mathbb{S}^{\mathfrak{n}}\right)$ satisfying

$$
z\langle\hat{u}(z), v\rangle+\langle\operatorname{grad} \hat{u}(z), \operatorname{grad} v\rangle=\langle g(z), v\rangle, \quad \text { for all } v \in \mathrm{H}^{1}\left(\mathbb{S}^{n}\right) .
$$


Our assumption (12) ensures that $V_{h} \subseteq H^{1}\left(\mathbb{S}^{n}\right)$, since $\tau>n / 2 \geqslant 1$. Applying the Galerkin method to (21), we seek $\widehat{u}_{h}(z) \in V_{h}$ satisfying

$$
z\left\langle\widehat{\mathfrak{u}}_{\mathrm{h}}(z), v\right\rangle+\left\langle\operatorname{grad} \hat{\mathfrak{u}}_{\mathrm{h}}(z), \operatorname{grad} v\right\rangle=\langle g(z), v\rangle, \quad \text { for all } v \in \mathrm{V}_{\mathrm{h}} .
$$

Concretely, to compute $\hat{\mathfrak{u}}_{\mathfrak{h}}(z)=\sum_{\mathrm{p}=1}^{\mathrm{K}} \mathrm{U}_{\mathrm{p}}(z) \Phi_{\mathrm{p}}$ we introduce the $\mathrm{K} \times \mathrm{K}$ mass matrix $B$ and stiffness matrix $S$, with entries

$$
\mathrm{B}_{\mathrm{pq}}=\left\langle\Phi_{\mathrm{q}}, \Phi_{\mathrm{p}}\right\rangle \text { and } \mathrm{S}_{\mathrm{pq}}=\left\langle\operatorname{grad} \Phi_{\mathrm{q}}, \operatorname{grad} \Phi_{\mathrm{p}}\right\rangle
$$

form the load vector $\mathbf{G}(z) \in \mathbb{C}^{\mathrm{K}}$ with components $\mathrm{G}_{\mathrm{p}}(z)=\left\langle\mathrm{g}(z), \Phi_{\mathrm{p}}\right\rangle$, and then solve the $\mathrm{K} \times \mathrm{K}$ complex linear system

$$
(z \mathrm{~B}+\mathrm{S}) \mathbf{U}(z)=\mathbf{G}(z),
$$

to obtain the solution vector $\mathbf{U}(z) \in \mathbb{C}^{\mathrm{K}}$ with components $\mathrm{U}_{\mathfrak{p}}(z)$.

\subsection{Fully-discrete solution}

Combining the time and space discretisations, we arrive at a fully discrete solution

$$
\mathrm{u}_{N, h}(\mathrm{t})=\frac{k}{2 \pi i} \sum_{j=-N}^{N} e^{z_{j} \mathrm{t}} \hat{u}_{h}\left(z_{j}\right) z_{j}^{\prime},
$$

whose evaluation requires that we solve the linear system (23) at each of the $2 \mathrm{~N}+1$ quadrature points $z_{\mathrm{j}}$. Moreover, in practice, we use quadratures for the integrations over $\mathbb{S}^{n}$ that are needed to compute $B_{p q}, S_{p q}$ and $G_{p}(z)$.

\section{$4 \quad$ Numerical experiments}

We describe two example problems. 


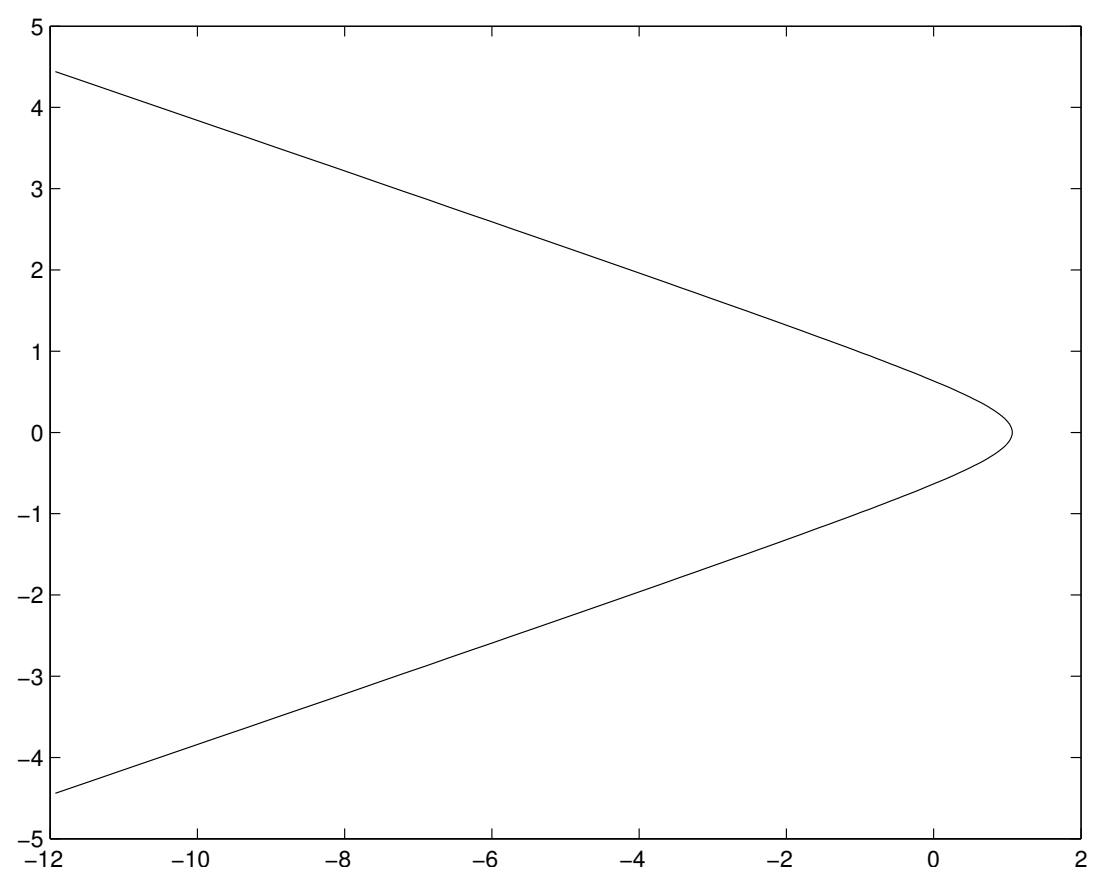

FigURE 1: Integration contour.

\subsection{A scalar problem}

For our first example, we take $A=1$, which reduces (1) to an ordinary differential equation, and choose the initial data and source term so that the exact solution is

$$
\mathrm{u}(\mathrm{t})=1+\frac{4 \mathrm{t}^{3 / 2}}{3 \sqrt{\pi}}
$$

In this way, $\hat{\mathfrak{u}}(z)=z^{-1}+z^{-5 / 2}$ and $\hat{\mathrm{f}}(z)=z^{-3 / 2}+z^{-1}+z^{-5 / 2}$, so we may choose an integration contour as in Figure 1, avoiding the branch cut along the negative real axis, with $\omega=1, \lambda=3 / 2$ and $\delta=\pi / 2-0.3$. The numerical solution is given by (18), because no space discretisation is required. In other words, the error arises solely from the quadrature rule. Table 2 shows the error 
TABLE 2: Quadrature errors.

\begin{tabular}{cccccc}
\hline $\mathrm{N}$ & 10 & 20 & 40 & 80 & 90 \\
\hline$\left|\mathrm{U}_{\mathrm{N}}(2)-\mathrm{u}(2)\right|$ & 0.05 & $5.0 \mathrm{E}-04$ & $1.1 \mathrm{E}-09$ & $6.2 \mathrm{E}-15$ & $2.2 \mathrm{E}-15$ \\
$\exp (-0.3793 \mathrm{~N})$ & 0.02 & $5.1 \mathrm{E}-04$ & $2.6 \mathrm{E}-07$ & $6.7 \mathrm{E}-14$ & $1.5 \mathrm{E}-15$ \\
\hline
\end{tabular}

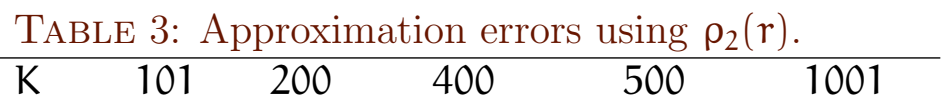

\begin{tabular}{ccccccc}
$\mathrm{N}$ & $\mathrm{h}_{\mathrm{X}}$ & 0.256 & 0.180 & 0.128 & 0.113 & 0.079 \\
\hline 20 & $\mathrm{e}_{\mathrm{h}}$ & 0.057 & 0.003 & $1.8 \mathrm{E}-04$ & $8.3 \mathrm{E}-05$ & $2.4 \mathrm{E}-05$ \\
& $\mathrm{EOC}$ & & 8.51 & 8.05 & 6.35 & 3.53 \\
& $\mathrm{e}_{\mathrm{N}}(2)$ & 0.023 & 0.001 & $5.6 \mathrm{E}-04$ & $5.3 \mathrm{E}-04$ & $5.1 \mathrm{E}-04$ \\
& $\mathrm{EOC}$ & & 8.30 & 2.25 & 0.45 & 0.12 \\
\hline 40 & $e_{h}$ & 0.057 & 0.003 & $1.8 \mathrm{E}-04$ & $8.3 \mathrm{E}-05$ & $2.4 \mathrm{E}-05$ \\
& EOC & & 8.51 & 8.05 & 6.35 & 3.49 \\
& $e_{N}(2)$ & 0.023 & 0.001 & $7.5 \mathrm{E}-05$ & $3.1 \mathrm{E}-05$ & $7.0 \mathrm{E}-06$ \\
& EOC & & 8.35 & 8.20 & 6.89 & 4.26 \\
\hline
\end{tabular}

at time $t=2$ for different values of $N$, using the quadrature step $k=3 / N$. The rapid convergence of the method is apparent. The rate of convergence is $\mathcal{O}\left(e^{-\mu N}\right)$ for some $\mu$ in the range of $[0.1,0.65]$, which is consistent with theoretical estimates in (20).

\subsection{A problem on the unit sphere}

We now take $A=-\Delta^{*}$ on the two-sphere $\mathbb{S}^{2}$, and choose $u_{0}$ and $f$ so that the exact solution of $(1)$ is

$$
u(x, t)=\sin x-\frac{4}{3 \sqrt{\pi}} t^{3 / 2} \sin (2 x)
$$

For the spatial discretisation, we use the compactly supported radial basis 
TABLE 4: Approximation errors using $\rho_{3}(r)$.

\begin{tabular}{ccccccc}
\hline & $\mathrm{K}$ & 101 & 200 & 400 & 500 & 1001 \\
$\mathrm{~N}$ & $\mathrm{~h}_{\mathrm{X}}$ & 0.256 & 0.180 & 0.128 & 0.113 & 0.079 \\
\hline 20 & $e_{\mathrm{h}}$ & 0.102 & 0.003 & $1.2 \mathrm{E}-04$ & $4.0 \mathrm{E}-05$ & $9.2 \mathrm{E}-07$ \\
& $\mathrm{EOC}$ & & 9.61 & 9.91 & 8.68 & 10.70 \\
& $e_{\mathrm{N}}(2)$ & 0.041 & 0.002 & $5.2 \mathrm{E}-04$ & $5.1 \mathrm{E}-04$ & $5.0 \mathrm{E}-04$ \\
& $\mathrm{EOC}$ & & 9.16 & 3.32 & 0.17 & 0.04 \\
\hline 40 & $e_{h}$ & 0.102 & 0.003 & $1.2 \mathrm{E}-04$ & $4.0 \mathrm{E}-05$ & $9.2 \mathrm{E}-07$ \\
& $\mathrm{EOC}$ & & 9.61 & 9.91 & 8.68 & 10.70 \\
& $e_{\mathrm{N}}(2)$ & 0.041 & 0.001 & $5.0 \mathrm{E}-05$ & $1.7 \mathrm{E}-05$ & $3.5 \mathrm{E}-07$ \\
& $\mathrm{EOC}$ & & 9.57 & 9.87 & 8.68 & 10.97 \\
\hline
\end{tabular}

functions from Table 1 , and to generate the set of points $X$ we use an equal area partitioning algorithm of Saff and Kuijlaars [5]. We compute the inner products arising in the matrix entries (22) and the load vector components $G_{p}(z)$ using a quadrature approximation of the form

$$
\int_{\mathbb{S}^{2}} v \approx \frac{2 \pi}{\mathrm{R}} \sum_{\mathrm{q}=1}^{\mathrm{R}} \sum_{\mathrm{p}=1}^{\mathrm{R} / 2} w_{\mathrm{p}} v\left(\sin \theta_{\mathrm{p}} \cos \phi_{\mathrm{q}}, \sin \theta_{\mathrm{p}} \sin \phi_{\mathrm{q}}, \cos \theta_{\mathrm{p}}\right)
$$

for an even number $R \geqslant 2$, where $\int_{-1}^{1} f(z) d z \approx \sum_{p=1}^{R / 2} w_{p} f\left(\cos \theta_{p}\right)$ is a GaussLegendre rule and $\phi_{\mathrm{q}}=2 \pi \mathrm{q} / \mathrm{R}$. The error in the approximation (24) is zero if the integrand $v$ is a polynomial of total degree $R-1$ or less.

Tables 3 and 4 show values of

$$
e_{h}=\max _{|j| \leqslant N}\left|\hat{u}_{h}\left(z_{j}\right)-\hat{u}\left(z_{j}\right)\right| \text { and } e_{N}(2)=\left|u_{N}(2)-u(2)\right|,
$$

for different choices of $\mathrm{K}$ and $\mathrm{N}$, along with the estimated order of convergence (EOC). The quantity $e_{h}$ is a measure of the error arising from the spatial discretisation, whereas $e(2)$ includes also the contribution from the time discretisation. 
Tables 3 and 4 show that the smoother the RBF the higher the convergence rate of the method. The accuracy of the method also depends on the accuracy of the quadrature used for time discretisation. Compared with a spectral method, our implementation is much simpler since we do not have to evaluate surface spherical harmonics. Compared with a finite element method, a triangular mesh for the sphere is not needed. The method allows a parallel implementation, and can be generalised easily to other classes of operators that satisfy the resolvent estimates.

Acknowledgements The support of the Australian Research Council is gratefully acknowledged.

\section{References}

[1] Debao Chen, Valdir A. Menegatto, and Xingping Sun. A necessary and sufficient condition for strictly positive definite functions on spheres. Proc. Amer. Math. Soc., 131:2733-2740, 2003. doi:10.1090/S0002-9939-03-06730-3 C94

[2] Q. T. Le Gia. Approximation of parabolic PDEs on spheres using spherical basis functions. Adv. Comput. Math., 22:377-397, 2005. doi:10.1007/s10444-003-3960-9 C90, C91

[3] William McLean and Vidar Thomée. Numerical solution via Laplace transforms of a fractional order evolution equation. J. Integral Equations Appl., 22:57-94, 2010. doi:10.1216/JIE-2010-22-1-57 C91, C96

[4] M. N. Özisik. Heat conduction. John Wiley \& Sons, Inc., New York, 1993. C90

[5] E. B. Saff and A. B. J. Kuijlaars. Distributing many points on a sphere. Math. Intelligencer, 19:5-11, 1997. doi:10.1007/BF03024331 C100 
[6] I. J. Schoenberg. Positive definite functions on spheres. Duke Math. J., 9:96-108, 1942. doi:10.1215/S0012-7094-42-00908-6 C93

[7] Dongwoo Sheen, Ian H .Sloan, and Vidar Thomée. A parallel method for time-discretization of parabolic equations based on contour integral representation and quadrature. Math. Comp., 69:177-195, 1999. doi:10.1090/S0025-5718-99-01098-4 C91

[8] Holger Wendland. Scattered Data Approximation. Cambridge University Press, Cambridge, 2005. doi:10.2277/0521843359 C94

[9] Yuan Xu and E. W. Cheney. Strictly positive definite functions on spheres. Proc. Amer. Math. Soc., 116:977-981, 1992. doi:10.1090/S0002-9939-1992-1096214-6 C93

\section{Author addresses}

1. Quoc Thong Le Gia, School of Mathematics and Statistics, University of New South Wales, Sydney, Australia. mailto:qlegia@unsw.edu.au

2. William McLean, School of Mathematics and Statistics, University of New South Wales, Sydney, Australia.

mailto:w.mclean@unsw. edu. au 